\title{
Electrostatic Control of Dual-Core Optical Fibre with NEMS Functionality
}

\author{
Nina Podoliak, Zhenggang Lian, Peter Horak, Wei H. Loh \\ Optoelectronics Research Centre, University of Southampton, Southampton, SO17 1BJ, United Kingdom
}

A nanomechanical optical fibre with a mechanically adjustable dual-core structure has been demonstrated recently [1]. The realisation of equivalent systems with an electric actuation would allow for low-loss all-fibre reconfigurable optical networks. Here we investigate the feasibility of nanoelectromechanical actuation of the dual-core fibre. Such optical systems with mechanically adjustable or controllable components are well known in the form of silicon micro- and nanoelectromechanical systems (MEMS and NEMS) and are widely used in various optoelectronic devices, for example 2-D optical switches and reconfigurable optical add-drop multiplexers.

The fibre consists of two elliptical cores that are independently suspended in a central hole of a glass fibre by thin glass membranes and can be moved with respect to each other, see Fig. 1a. The optical properties of the fibre are sensitive to even nanometre displacements of the cores and can therefore be controlled very accurately by minute external forces. By changing the separation gap between the cores it is possible to adjust effective mode indices and to change optical coupling lengths, which can lead to a wide range of applications in the fields of sensing, switching and all-optical network control.

Different methods to move the cores in the dual core fibre have been proposed. An optical switch based on applying gas pressure has been demonstrated [1], and optomechanical forces have been investigated theoretically. However, for most applications a faster and more robust actuation method will be required. Here we investigate the deflection of the cores by the electrostatic force: a non-uniform electric field is used to induce dipole forces on the fibre cores and pull them towards the region of stronger electric fields. In order to achieve a large field gradient, metallic wire electrodes need to be introduced in the vicinity of the optical cores [2]. The induced displacement of the cores is then proportional to the square of the voltage applied to the electrodes.

We model the optical and mechanical properties, such as phase and group velocity of different modes, dispersion, and switching speed for a range of various fibre geometries, including configurations with one to four wire electrodes, Fig. 1b. Taking into account realistic sizes for the cores $(2 \times 0.8 \mu \mathrm{m})$ and the supporting membrane $(0.2 \mu \mathrm{m}$ thick and $20 \mu \mathrm{m}$ wide, Fig. 1a) and assuming 50 Volts applied to the electrodes, the relative movement of the cores depending on their initial separation is calculated using a Finite Element Method (Comsol Multiphysics ${ }^{\circledR}$ ) (Fig.1c). Of the fibre geometries shown here the quadrupole (four wire) configuration yields the largest field gradient and therefore the largest forces to pull the cores apart. Core displacements greater than $0.5 \mathrm{~nm}$ are predicted at 50 Volts applied to the electrodes. Such a variation of the geometry changes the beat length of the coupled core modes and thus, for example, the output at the fibre end can be switched continuously between the two cores. For a fibre of only $15 \mathrm{~cm}$ length with an initial core-to-core separation of $1.5 \mu \mathrm{m}$, a complete switch of the light from one core to another is predicted at $\sim 30 \mathrm{~V}$. We are currently working on the fabrication and experimental demonstration of such a fibre and its NEMS functionality.

(a)

(b)

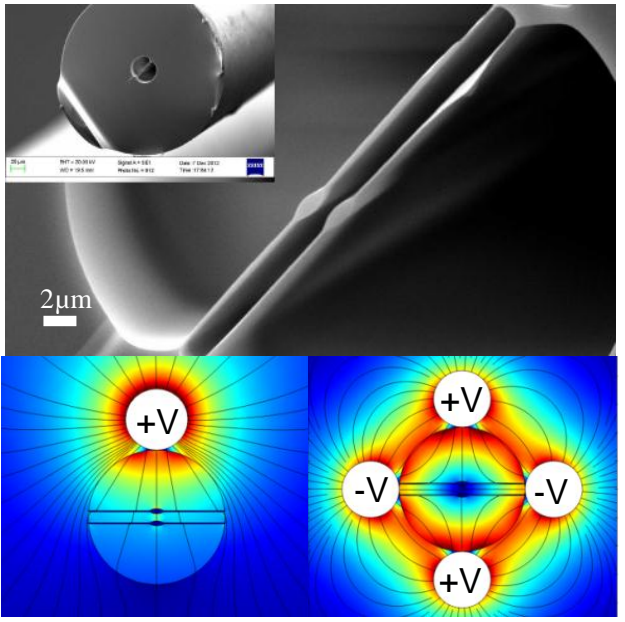

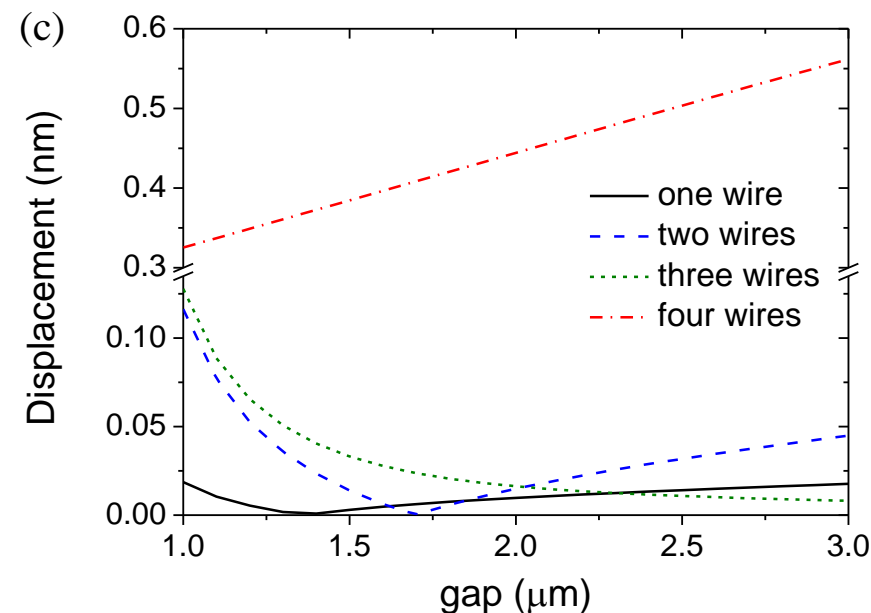

Fig. 1 (a) SEM photograph of a fabricated dual-core optical fibre. (b) Electric field distributions for different wire geometries. (c) Relative core displacement depending on the initial core-to-core separation for different fibre geometries. In all calculations $\mathrm{V}=50$ Volts.

\section{References}

[1] Z. Lian, P. Horak, X. Feng, L. Xiao, K. Frampton, N. White, J. A. Tucknott, H. Rutt, D. N. Payne, W. Stewart, and W. H. Loh, "Nanomechanical optical fiber," Opt. Express 20, 29386-29394 (2012).

[2] M. Bayindir, F. Sorin, A.F. Abouraddy, J. Viens, S.D. Hart, J.D. Joannopoulos and Y. Fink. "Metal-insulator-semiconductor optoelectronic fibres", Nature 431, 826-829 (2004). 